\title{
The Changing Face of Head and Neck Cancer in the 21st Century: The Impact of HPV on the Epidemiology and Pathology of Oral Cancer
}

\author{
William H. Westra
}

Received: 2 January 2009/Accepted: 11 January 2009/Published online: 24 February 2009

(C) Humana 2009

\begin{abstract}
The longstanding notion that head and neck squamous cell carcinoma (HNSCC) is a uniform disease process is changing. Divergence in epidemiologic trends among HNSCCs arising in different anatomic subsites has introduced a view that HNSCC is a heterogeneous group. Analysis of molecular genetic changes discloses not just individual tumor differences, but also consistent large-scale differences that permit the recognition of important tumor subtypes. One recently recognized subtype is the human papillomavirus (HPV)-positive oropharyngeal carcinoma. HPV-positive oropharyngeal cancer now dominates the head and neck oncology landscape, and its escalating incidence is impacting on diagnostic, preventive and therapeutic practices.
\end{abstract}

Keywords Human papillomavirus - Oral cancer . Head and neck squamous cell carcinoma .

In situ hybridization

\section{Introduction}

Long regarded as a uniform group of tumors that differed only as a function of anatomic site, ongoing studies indicate that HNSCCs may not be as homogenous as previously supposed. Recognition of distinct molecular genetic profiles now permits finer resolution of HNSCC

\footnotetext{
W. H. Westra $(\square)$

Department of Pathology and Otolaryngology/Head \& Neck Surgery, The Johns Hopkins Medical Institutions, The Weinberg Bldg, Room 2242, 401 N, Broadway, Baltimore,

MD 21231, USA

e-mail: wwestra@jhmi.edu
}

into distinct subtypes that differ with respect to risk factors, pathogenesis, and clinical behavior [1]. HPV-associated oropharyngeal carcinoma has recently been recognized as a unique subtype of HNSCC [2]. HPV status has a profound effect on patient prognosis, and it may soon guide therapy. Accordingly, HPV status will become a standard component in the diagnostic reporting of all oropharyngeal carcinomas. This review covers recent advances toward defining HPV-related oropharyngeal carcinoma, and draws specific attention to pathology concerns that impact on the diagnosis and reporting of these cancers.

\section{Discussion}

HNSCC is the eighth most common cancer worldwide with approximately 650,000 new cases reported annually. Beginning in the early 1980s, the incidence of HNSCC in the United States and elsewhere has been in retreat. This downward trend has roughly paralleled trends in smoking. Accordingly, HNSCC is often perceived as a preventable disease, and its ultimate demise simply a matter of implementing behavior modification strategies. This great optimism has recently been shaken by a sobering escalation of a specific type of cancer that is asserting its dominance on the head and neck oncology landscape. Specifically, the incidence of oropharyngeal cancer is advancing [3, 4]. This anomalous rise bucks the prevailing notion that HNSCC incident rates can be controlled through smoking prevention and cessation. Indeed, the escalation of oropharyngeal carcinoma points to the participation of non-traditional behavioral and environmental factors driving this disturbing epidemiologic trend. 
The prototypic patient with oral cancer is an older man who has smoked cigarettes and drank alcohol for many years. This prototype, however, no longer fits the patient with oral cancer who now enters the doors of our clinics and hospitals. Patients now tend to be younger (between 40 and 60 years) white men who have never smoked cigarettes or drank alcohol [5]. Traditional risk factors have been supplanted by other powerful risk factors relating to sexual practices, the most important of these being high number of sexual partners, history of oral-genital sex, and history of oral-anal sex $[5,6]$. Oral anogenital contact is an important route for the transmission of HPV to the oral cavity. Certain conditions and behaviors that alter antitumor immunity may be important in transforming an HPV oral infection towards HPV-related malignancy. As one example, marijuana use has recently been identified as an independent risk factor for HPV-positive HNSCC, and the strength of this association increases with intensity, duration, and cumulative years of marijuana smoking [7]. Long scrutinized as a potential source of DNA-damaging carcinogens, marijuana smoke may be more relevant for its immunomodulatory effects. Cannabinoids bind to the $\mathrm{CB} 2$ receptor expressed on immunomodulatory cells in human tonsillar tissue. Binding, in turn, can suppress immune responses, diminish host responses to viral pathogens, and attenuate antitumor activity.

HPV, particulary type 16 , is detected in about $70 \%$ of oropharyngeal carcinomas. These HPV-positive cancers are increasingly recognized as a distinct subgroup of HNSCC with a biological and clinical profile that diverges from that of their HPV-negative counterparts (Table 1). At the molecular genetic level, HPV-positive HNSCCs express the viral oncoproteins E6 and E7, overexpress the p16 gene product, and only infrequently harbor p53 gene mutations. Regarding clinical behavior, HPV16-positive HNSCs are associated with an improved prognosis [8, 9]. The mechanisms underlying this favorable prognosis may involve the combined effects of immune surveillance to viral-specific tumor antigens, an intact apoptotic response to radiation, and the absence of widespread genetic alterations associated with smoking (i.e., field cancerization).
Table 1 Comparison of HPV-positive and HPV-negative head and neck cancers

\begin{tabular}{|c|c|c|}
\hline & HPV-positive & HPV-negative \\
\hline Incidence & Increasing & Decreasing \\
\hline Age & Younger & Older \\
\hline Gender & $3: 1$ men & $3: 1$ men \\
\hline Risk factors & Sexual behavior & $\begin{array}{l}\text { Tobacco, } \\
\text { alcohol }\end{array}$ \\
\hline Cofactors & $\begin{array}{l}\text { Marijuana, } \\
\text { immunosuppression }\end{array}$ & Diet, oral hygiene \\
\hline \multirow{3}{*}{$\begin{array}{l}\text { Molecular genetics } \\
\text { findings }\end{array}$} & $\mathrm{P} 16 \uparrow$ & $\mathrm{P} 16 \downarrow$ \\
\hline & $\mathrm{Rb} \downarrow$ & $\mathrm{Rb} \uparrow$ \\
\hline & P53 wild-type & P53 mutated \\
\hline Anatomic site & Lingual and palatine tonsils & All sites \\
\hline \multicolumn{3}{|l|}{ Pathologic findings } \\
\hline Primary & Basaloid & Keratinized \\
\hline $\begin{array}{r}\text { Lymph node } \\
\text { metastasis }\end{array}$ & Cystic & Solid \\
\hline Survival & Better & Worse \\
\hline
\end{tabular}

The pathologic features of HPV-positive HNSCs also deviate from the moderately differentiated keratinizing morphology that typifies most HNSCCs. HPV-positive HNSCCs consistently: (1) arise from the tonsillar crypts; (2) are unassociated with dysplasia of the surface epithelium; (3) exhibit lobular growth; (4) are permeated by infiltrating lymphocytes; (5) lack significant keratinization; and (6) demonstrate a prominent "basaloid" morphology (Fig. 1). Two microscopic features of HPV-related oropharyngeal cancers are likely to cause diagnostic ambiguity. First, HPV-related HNSCC is customarily misperceived as a poorly differentiated carcinoma based on the immature appearance of the tumor cells. In point of fact, the appearance of the tumor cells closely emulates the appearance of the reticulated epithelium - the specialized epithelium lining the tonsillar crypts from which HPV-related cancers arise [10]. In other words, HPV-related oropharyngeal cancers are in fact highly differentiated, not poorly
Fig. 1 Typical histopathologic appearance of an HPV-related cancer of the head and neck. a The carcinomas tend to arise from the tonsillar crypts and infiltrate the lymphoid stroma as expanding tumor lobules. b The lobules of tumor cells are permeated by lymphocytes, lack keratinization, and have a basaloid appearance
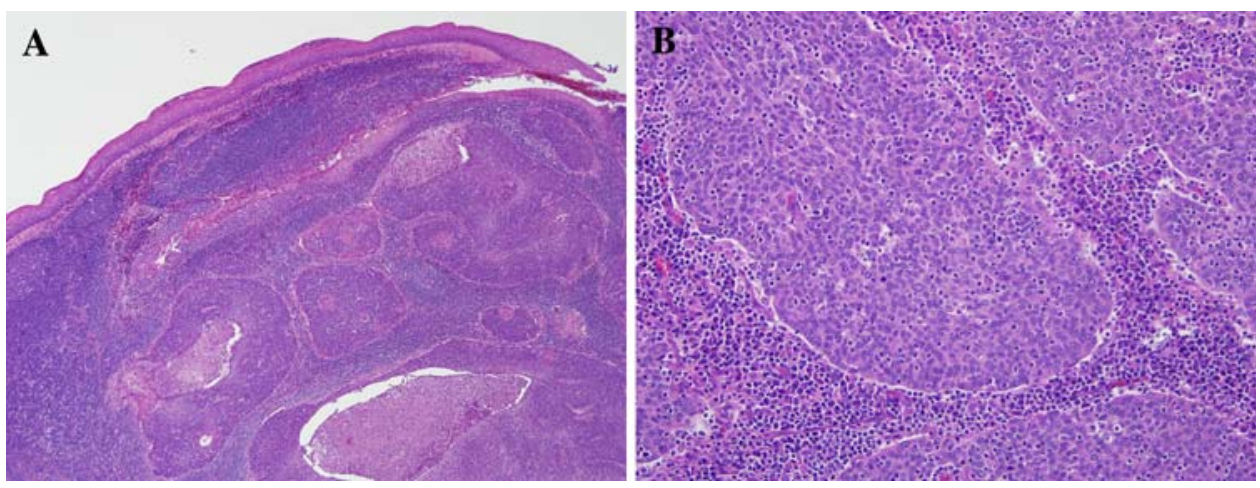
differentiated as so widely assumed. Second, use of the term "basaloid" as a diagnostic descriptor is confusing for the way it invites an erroneous connection with basaloid squamous cell carcinoma-a subtype of HNSCC notorious for its aggressive clinical behavior. Within the basaloid subtype, detection of HPV is a highly favorable prognostic factor that helps identify a subset of cancers that departs from the highly aggressive behavior associated with this variant [11]. Until consensus panels put forward a classification scheme for oropharyngeal carcinomas that underscores their relationship with HPV while avoiding confusion with the aggressive basaloid variant, it is our practice to: (1) classify these tumors as non-keratinizing squamous cell carcinomas, (2) suspend the use of the descriptors such as "poorly differentiated" and "basaloid", and (3) routinely report on the HPV status of all HNSCCs arising in the oropharynx.

Determination of HPV status will certainly become standard practice in the pathologic evaluation of oropharyngeal cancers. As a biomarker, HPV detection is emerging as a valid method of discerning the presence and progress of disease encompassing all aspects of patient care from early cancer detection, to tumor localization [12] (Fig. 2), to selection of patients most likely to benefit from specific therapies, to post-treatment tumor surveillance. A variety of detection methods are in current use including PCR-based strategies, type-specific in situ hybridization (ISH) techniques, and immunohistochemical detection of surrogate biomarkers (e.g. p16 protein). Standardization of HPV detection in the clinical arena must begin with selection of the best detection platform for universal application.

The preferential use of ISH methods over PCR-based methods is supported both by biological and practical considerations. The reported large variation of HPV prevalence in HNSCC is a largely a reflection of the inability of non-quantitative PCR methods to discern virus that is biologically meaningful from virus that is biologically irrelevant. In contrast, punctuate hybridization signals within the nuclei of tumor cells is a pattern of staining only seen following HPV DNA integration into the host genome, and thus is more closely linked with relevant viral infections. Importantly, the improved specificity of HPV detection by ISH does not come at the expense of sensitivity. The introduction of various signal amplification steps has significantly improved the sensitivity of this technique, even to the point of viral detection down to one viral copy per cell. The development of nonfluorescent chromogens now allows visualization of DNA hybridization using conventional light microscope. Adaptation of ISH to formalin-fixed and paraffin-embedded tissues has made this technique compatible with standard tissue-processing procedures and amendable to retrospective analysis of archival tissue blocks while most PCR-based methods are optimized to fresh frozen samples. ISH is a feasible and cost-effective test for most diagnostic laboratories that routinely process formalin-fixed and paraffin-embedded tissue blocks.

In HPV-positive oropharyngeal carcinomas, functional inactivation of $\mathrm{Rb}$ by the viral oncoprotein E7 is known to induce an up regulation of p16 expression, reaching levels that can be readily detected by routine immunohistochemistry. Accordingly, p16 immunohistochemistry is often advocated as a reliable surrogate marker of HPVinduced neoplasia of oropharynx. Direct comparison of p16 immunohistochemical staining and HPV-16 ISH for large numbers of HNSCCs reveals a discrepancy rate of about $25 \%$. In a subset of discrepant cases, high p16 expression is due to the presence of some other (non-16) HPV type as confirmed by wide spectrum ISH. The remaining discrepancies likely reflect the imperfection of p16 as a surrogate marker. Using E6/E7 mRNA levels as conclusive evidence of HPV involvement, positive p16 immunostaining of HNSCCs is $100 \%$ sensitive but only $79 \%$ specific [13].

The limitations of any single detection assay may be offset using algorithms that combine the strengths of complementary assays. We use a detection strategy that combines HPV ISH with p16 immunohistochemistry. Given a sensitivity that approaches $100 \%$, p16 immunostaining is a good first line assay for eliminating HPVnegative cases from additional analysis. HPV-16 ISH can
Fig. 2 Metastatic squamous cell carcinoma of unknown primary origin. The metastasis is cystic (a, hematoxylin and eosin). The presence of HPV as evident by $\mathrm{p} 16$

immunohistochemical staining (b) and HPV-16 in situ hybridization (hybridization dots within tumor cell nuclei, inset) point to the oropharynx as the site of tumor origin
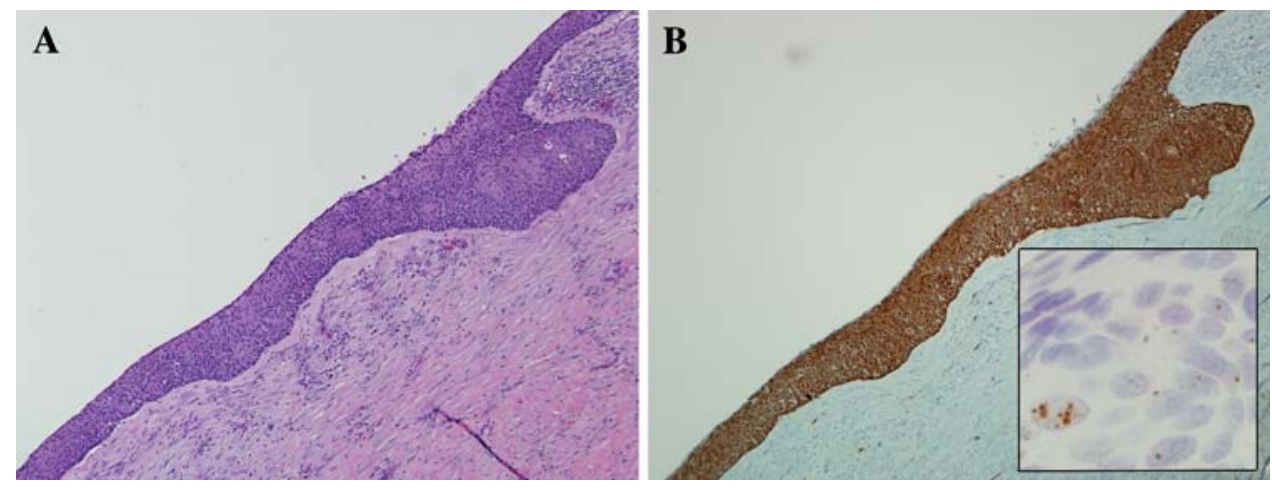
be run concurrently with p16 immunostaining or as a second-line assay following a positive p16 result. Given a specificity approaching $100 \%$, a positive HPV-16 ISH reduces the numbers of false positive cases by p16 staining alone. A p16-positive/HPV-16-negative result singles out a subset of tumors that qualify for rigorous analysis for other (i.e. non-16) oncogenic HPV types. For this third-line assay, we use a consensus ISH probe that that detects an extended panel of HPV types. P16 immunohistochemistry and HPV ISH are standardized techniques that are easily applied to formalin-fixed and paraffin-embedded tissues. As automated ISH technologies are brought on-line, turnaround time will be further shortened and standardization across various diagnostic laboratories will be enhanced.

\section{Conclusion}

Incident trends for HNSCC have generally paralleled smoking trends. The escalating incidence of oropharyngeal carcinoma in the absence of a parallel rise in smoking and alcohol consumption suggests that nontraditional behavioral and environmental factors are driving this aberration. HPV, particularly type 16, has been established as a causative agent in up to $70 \%$ of oropharyngeal cancers. These HPV-positive HNSCCs differ in important respects from HPV-negative HNSCC including risk factors, molecular genetic alterations, microscopic appearance, and clinical behavior. Diagnostic pathologists are now faced with the challenge of accurately discerning HPV status of oropharyngeal cancers.

\section{References}

1. Pai SI, Westra WH. Molecular pathology of head and neck cancer: implications for diagnosis, prognosis and treatment of patients with head and neck squamous cell carcinoma. Annual Rev Pathol. 2008 (Epub ahead of print).
2. Gillison ML, Koch WM, Capone RB, Spafford M, Westra WH. Evidence for a causal association between human papillomavirus and a subset of head and neck cancers. J Natl Cancer Inst. 2000;92:709-20.

3. Sturgis EM, Cinciripini PM. Trends in head and neck cancer incidence in relation to smoking prevalence: an emerging epidemic of human papillomavirus-associated cancers? Cancer. 2007;110:1429-35.

4. Chaturvedi AK, Engels EA, Anderson WF, Gillison ML. Incidence trends for human papillomavirus-related and-unrelated oral squamous cell carcinomas in the United States. J Clin Oncol. 2008;26:612-9.

5. D'Souza G, Kreimer AR, Viscidi R, Pawlita M, Fakhry C, et al. Case-control study of human papillomavirus and oropharyngeal cancer. N Engl J Med. 2007;356:1944-56.

6. Smith EM, Ritchie JM, Summersgill KF, Klussmann JP, Lee JH, et al. Age, sexual behavior and human papillomavirus infection in oral cavity and oropharyngeal cancers. Int J Cancer. 2004;108: 766-72.

7. Gillison ML, D’Souza G, Westra WH, Sugar E, Xiao W, et al. Distinct risk factor profiles for human papillomavirus-16 positive and negative head and neck cancer. J Natl Cancer Inst. 2008;100: 407-20.

8. Fakhry C, Westra WH, Li S, Cmelak A, Ridge JA, et al. Improved survival of patients with human papillomaviruspositive head and neck squamous cell carcinoma in a prospective clinical trial. J Natl Cancer Inst. 2008;100:261-9.

9. Weinberger PM, Yu Z, Haffty BG, Kowalski D, Harigopal M, et al. Molecular classification identifies a subset of human papillomavirus-associated oropharyngeal cancers with favorable prognosis. J Clin Oncol. 2006;24:736-47.

10. Begum S, Cao D, Gillison M, Zahurak M, Westra WH. Tissue distribution of human papillomavirus 16 DNA integration in patients with tonsillar carcinoma. Clin Cancer Res. 2005;11: 5694-9.

11. Begum S, Westra WH. Basaloid squamous cell carcinoma of the head and neck is a mixed variant that can be further resolved by HPV status. Am J Surg Pathol. 2008;32:1044-50.

12. Begum S, Gillison ML, Ansari-Lari MA, Shah K, Westra WH. Detection of human papillomavirus in cervical lymph nodes: a highly effective strategy for localizing site of tumor origin. Clin Cancer Res. 2003;9:6469-75.

13. Smeets SJ, Hesselink AT, Speel EJ, Haesevoets A, Snijders PJ, Pawlita M, et al. A novel algorithm for reliable detection of human papillomavirus in paraffin embedded head and neck cancer specimen. Int J Cancer. 2007;121:2465-72. 